\title{
Born Inversion for Broadband Ultrasonic Monitoring of Cancer Treatment
}

\author{
Basak Ulker Karbeyaz*, Eric L. Miller ${ }^{\dagger}$, Robin O. Cleveland ${ }^{* *}$ and \\ Ronald A. Roy* \\ ${ }^{*}$ Analogic Corporation, Peabody, MA 01960 \\ ${ }^{\dagger}$ Northeastern University, Boston MA 02115 \\ ** Boston University, Boston MA 02115
}

\begin{abstract}
One barrier to developing High Intensity Focused Ultrasound (HIFU) methods for clinical use is monitoring the progress of the treatment by non-invasive imaging techniques. In this work, we propose the use of ultrasound-based imaging techniques to characterize the geometric structure of the HIFU lesion. To obtain quantitatively accurate reconstructions of the HIFU anomaly, one could invert for the acoustic properties on a densely sampled grid of voxels. However, the computational size of the problem makes traditional pixel based inversion methods impractical. Hence, we exploit the fact that HIFU results in approximately ellipsoidal lesions in which sound speed and attenuation are altered from their nominal values. By employing shape-based methods it is only necessary to estimate a small number of parameters to describe the geometry of the lesion. The details of this nonlinear inversion method are provided and its performance and robustness are demonstrated using broadband ultrasound backscatter data obtained with a commercial ultrasound scanner and a tissue phantom containing a HIFU-like lesion.
\end{abstract}

Keywords: Ultrasound, HIFU, Born approximation, Shape based inversion, Ellipsoid

\section{INTRODUCTION}

Traditional medical ultrasound scanners employ beamforming to generate 2-D images in real time. These techniques have enjoyed a great deal of success both because of their inherent computational efficiency as well as the quality of the imagery they provide. Less common in practice are image formation methods based on the underlying wavefield physics of the ultrasound sensor. Though capable of providing quantitatively accurate information concerning the state of the tissue, these model-based approaches are challenging because of the ill-posed nature of the associated inverse problem as well as the computational burden that arises with the required discretization of the physical model.

In this paper we present the application of wave-based image formation that overcomes these difficulties in the context of ultrasound-based image-guided cancer treatment using high intensity focused ultrasound (HIFU). We used ultrasound imaging techniques to characterize the geometric structure and acoustic properties, specifically the sound speed, attenuation and density, of the HIFU lesion.

The computational size of the relevant 3D ultrasound problem renders impractical the use of nonlinear inversion methods. Thus we rely on a linearized model, the well-known first Born approximation, as the basis for determining both the perturbations in the three acoustic parameters, and the background properties around which the linearization was 
performed.

To obtain quantitatively accurate reconstructions of the HIFU anomaly, one could invert for the acoustic properties on a densely sampled grid of voxels. However, the size of the problem makes traditional pixel based inversion methods unfeasible. In this study we developed and implemented a shaped based inversion method and, rather than trying to reconstruct the thousands of voxels defining the lesion, we concentrated on specifying the parameters describing the shape of the perturbation. The results demonstrate that the shape based inversion method can be employed for ultrasound data. The proposed method is validated using measured broadband ultrasound backscatter data.

\section{FORWARD MODEL}

The propagation of time harmonic ultrasound through an inhomogeneous, lossy medium can be described by the following frequency domain wave equation $[1,2]$ :

$$
\nabla^{2} p(r, \omega)-\nabla \ln \rho(r) \cdot \nabla p(r, \omega)+k^{2}(r, \omega) p(r, \omega)=q(r, \omega)
$$

where $r$ is a point in 3-space, $\omega$ is the angular frequency, $p(r, \omega)$ is the pressure at $r$ and $\omega, \rho(r)$ is the density, $c(r)$ is the speed of sound, $k(r, \omega)$ is the wavenumber and $q(r, \omega)$ is the source that creates the acoustic disturbance in the medium. In dispersive attenuating medium, $k(r, \omega)$ has the form : $k(r, \omega)=\frac{\omega}{c(r, \omega)}-j \alpha(r, \omega)$ where $c(r, \omega)$ is the dispersive speed of sound and $\alpha(r, \omega)$ is the frequency dependent attenuation coefficient.

In most tissue, the attenuation follows a power law, in which case the wavenumber can be expressed as [2]:

$$
k^{2}(r, \omega) \approx \frac{\omega^{2}}{c_{b}^{2}}-j \frac{2 \omega \alpha_{b}(\omega)}{c_{b}}-c_{p}(r)\left[\frac{2 \omega^{2}}{c_{b}^{3}}\right]-\alpha_{p}(r, \omega)\left[j \frac{2 \omega}{c_{b}}\right]
$$

where $c_{b}$ and $\alpha_{b}(\omega)$ are the speed of sound and attenuation in the tissue, $c_{p}(r)$ and $\alpha_{p}(r, \omega)$ are the perturbations to the sound speed and attenuation profiles respectively. The form of attenuation for power law dependency is: $\alpha_{b}(\omega)=\alpha_{b o}\left(\frac{\omega}{\omega_{o}}\right)^{n}$ and $\alpha_{p}(r, \omega)=\alpha_{p o}(r)\left(\frac{\omega}{\omega_{o}}\right)^{n}$ where typically $1<n<1.5$ for soft tissue . We invoke the Born approximation to determine the received echo signal in terms of the perturbation to the background properties [3]. After some algebraic manipulations the echo signal is given by [2]:

$$
p_{s}(r, \omega)=\int_{V^{\prime}}\left\{G\left(r, r^{\prime}, \omega\right) k_{s}^{2}\left(r^{\prime}, \omega\right) p_{b}\left(r^{\prime}, \omega\right)+\sigma_{p}\left(r^{\prime}\right) \nabla p_{b}\left(r^{\prime}, \omega\right) . \nabla G\left(r, r^{\prime}, \omega\right)\right\} d^{3} r^{\prime} .
$$

where $p_{s}(r, \omega)$ is the measured data (i.e. the Fourier transform of the ultrasonic echoes), $\sigma_{p}$ is the perturbation to the logarithm of the density, $p_{b}\left(r^{\prime}, \omega\right)$ is the background field; that is, the ultrasound field in the medium in the absence of the HIFU lesion, $G\left(r, r^{\prime}, \omega\right)$ is the 3D free space lossy Green's function and $V^{\prime}$ is boundary of the scattering volume. For convenience, Eq.(3) can be cast in the following compact form:

$p_{s}(r, \omega)=\int_{V^{\prime}} K_{1}\left(r, r^{\prime}, \omega\right) c_{p}\left(r^{\prime}\right) d^{3} r^{\prime}+\int_{V^{\prime}} K_{2}\left(r, r^{\prime}, \omega\right) \alpha_{p}\left(r^{\prime}\right) d^{3} r^{\prime}+\int_{V^{\prime}} K_{3}\left(r, r^{\prime}, \omega\right) \sigma_{p}\left(r^{\prime}\right) d^{3} r^{\prime}$ 
where $K_{i}, i=1,2,3$ depend only on the background properties.

For the imaging system of interest in this work we assume we have multiple transmitters and receivers. For the case of $N_{T}$ transmitters and $N_{R}$ receivers with $N_{S}$ frequency samples the measured data $(\mathbf{y})$ can be written as a column vector of size $N_{T} \mathrm{x} N_{S} \mathrm{x} N_{R}$. Discretizing Eq.(4) at any position $r^{\prime}$, the scattered field can be written in a matrix-vector model of the form:

$$
\mathbf{y}=\left[\begin{array}{lll}
\mathbf{K}_{\mathbf{1}} & \mathbf{K}_{\mathbf{2}} & \mathbf{K}_{\mathbf{3}}
\end{array}\right]\left[\begin{array}{lll}
\mathbf{f}_{\mathbf{1}}^{\mathrm{T}} & \mathbf{f}_{\mathbf{2}}^{\mathrm{T}} & \mathbf{f}_{\mathbf{3}}^{\mathrm{T}}
\end{array}\right]^{\mathrm{T}}+\mathbf{n}=\mathbf{K} \mathbf{f}+\mathbf{n}
$$

where $\mathbf{K}$ is the matrix representation of the integral, $\mathbf{f}_{\mathbf{1}}$ is the column vector containing the voxel values of sound speed perturbation, $c_{p}\left(r^{\prime}\right), \mathbf{f}_{\mathbf{2}}$ is the column vector containing the voxel values of attenuation perturbation, $\alpha_{p}\left(r^{\prime}\right), \mathbf{f}_{\mathbf{3}}$ is the column vector containing the voxel values of perturbation to the logarithm of the density, $\sigma_{p}\left(r^{\prime}\right)$ and $\mathbf{n}$ is the additive sensor noise.

\section{ELLIPSOID MODEL}

Under the Born approximation, the scatterer is viewed as a small perturbation to a homogenous background model and the model that we use for $\mathbf{f}_{\mathbf{i}}$ is the one where we have a constant perturbation of the parameter over an ellipsoidal shaped region.

A point $r^{\prime}=\left[x^{\prime}, y^{\prime}, z^{\prime}\right]^{T}$ in the region of interest is determined to be on or in the ellipsoid if $\left\|D_{i} U_{i}^{T}\left(r^{\prime}-c_{i}\right)\right\|_{2}^{2} \leq 1$ where $c$ is a length-3 vector, denoting the center of the ellipsoid, $\left(x_{o}, y_{o}, z_{o}\right), D=\operatorname{Diag}(d)$ is a diagonal matrix whose diagonal entries are the one over the lengths of the semi-axes, $\left(l_{1}, l_{2}, l_{3}\right)$, and $\mathrm{U}$ is an orthonormal matrix whose three columns are the coordinates of the semi-axes defined in terms of the Euler angles, $\left(\theta_{1}, \theta_{2}, \theta_{3}\right)$.

This condition can be imposed by multiplying $\left\|D_{i} U_{i}^{T}\left(r^{\prime}-c_{i}\right)\right\|_{2}^{2}$ by a support function $S\left(r^{\prime}\right)$ which has value unity in the ellipsoid and zero outside. Note that this formulation for $S\left(r^{\prime}\right)$ is a step function which is discontinuous at the boundary of the ellipsoid. The optimization routine requires a smooth approximation for the boundary. Specifically, the key to any non-linear minimization technique is to specify the Jacobian of the objective function with respect to the unknown parameters. With such a representation, the Jacobian must be evaluated numerically, thereby increasing computation time. To obtain an analytical representation, a differentiable expression is introduced to describe the boundary of the ellipsoids. Specifically, the perturbations in Eq.(4), $c_{p}\left(r^{\prime}\right), \alpha_{p}\left(r^{\prime}\right)$, and $\sigma_{p}\left(r^{\prime}\right)$ are represented with the following function:

$$
\gamma\left(r^{\prime}\right)=\exp \left(\tilde{M} \operatorname{sech}\left(1.3\left\|D U^{T}\left(r^{\prime}-c\right)\right\|_{2}^{16}\right)\right)-1
$$

where $\tilde{M}$ is a constant, related to the actual contrast $M$ with $\tilde{M}=\ln (M+1)$.

The parameters that will be used to characterize the ellipsoids of the three acoustic properties will be $\beta_{i}=\left[x_{o i}, y_{o i}, z_{o i}, l_{1 i}, l_{2 i}, l_{3 i}, \theta_{1 i}, \theta_{2 i}, \theta_{3 i}, M_{i}\right]$ where the subscripts $i=c, a, d$ will be used to represent the perturbations in sound speed, attenuation and density respectively. The representation of the perturbations given in Eq.(6), $\gamma\left(r^{\prime}\right)$, will be replaced by $\gamma_{i}\left(r^{\prime}, \beta_{i}\right)$ to account for the parameters of each individual ellipsoid. Mathematically speaking,

$$
\gamma_{i}\left(r^{\prime}, \beta_{i}\right)=\exp \left(\tilde{M}_{i} \operatorname{sech}\left(1.3\left\|D_{i} U_{i}^{T}\left(r^{\prime}-c_{i}\right)\right\|_{2}^{16}\right)\right)-1
$$


Given the data $\mathbf{y}$, we solve the nonlinear least squares problem:

$$
\min _{\beta_{c}, \beta_{a}, \beta_{d}}\|\mathbf{y}-\mathbf{K f}(\beta)\|_{2}^{2} \text { where } \beta=\left[\begin{array}{lll}
\beta_{c} & \beta_{a} & \beta_{d}
\end{array}\right]^{\mathrm{T}}
$$

We solve this minimization problem using the Gauss-Newton method [4].

\section{INVERSION PROCESS}

Using experimental data, we have verified the performance of the inversion approach for a case where density was not assumed to vary and the ellipsoids for attenuation and sound speed were co-located. An ellipsoidal target phantom (20mm x $5 \mathrm{~mm} \times 6 \mathrm{~mm}$ ) was carved from an agar-graphite sample. The acoustical parameters for this material were known to be $c=1545 \mathrm{~m} / \mathrm{s}, \rho=1045 \mathrm{~kg} / \mathrm{m}^{3}, \alpha=54\left(\frac{\omega}{\omega_{o}}\right)^{1.2} \mathrm{~Np} / \mathrm{m}$ where $\omega_{o}$ is the mean angular frequency $(2 \pi 3.5 \mathrm{MHz})$ [3]. The ellipsoid was imbedded in an optically transparent, cylindrically shaped (radius: $32.5 \mathrm{~mm}$, height: $125 \mathrm{~mm}$ ), polyacrylamide hydrogel with 7\% Bovine Serum Albumin (BSA) protein concentration. The acoustic properties of the background host phantom were: $c_{b}=1540 \mathrm{~m} / \mathrm{s}, \rho_{b}=1045 \mathrm{~kg} / \mathrm{m}^{3}, \alpha_{b}=$ $1.5 \mathrm{~Np} / \mathrm{m} / \mathrm{MHz}$ [5]. The BSA phantom had the same density as the agar phantom, which yielded a single ellipsoidal anomaly with sound speed and attenuation values compared to the background.

The transducer array (Model 8665, BK Medical, Wilmington, MA) consisted of 128 cylindrically curved elements, each of which was controlled individually by an Analogic AN2300 ultrasound imaging system (Analogic Corporation, Peabody, MA). The array was mechanically scanned across the ellipsoid anomaly at five measurement points. At each measurement point, a single element insonified the medium, and the response was recorded on five elements of the linear array. A Fourier transform of each of these 25 observed waveforms was performed, and five complex values frequency domain measurements per waveform were provided to the inversion algorithm.

In Fig. 1 (a)-(b) we display the the initial guess provided to our estimation method and the final estimate. For comparison purposes, the expected shape of the target is plotted in blue in Fig. 1(c). The total computation time was about 26 minutes using Matlab on a Pentium-class PC. The quantitative acoustic parameters of the ellipsoid were also estimated to a high degree of accuracy. Starting from an initial guess of $20 \mathrm{~m} / \mathrm{s}$ for the sound speed perturbation, the Gauss-Newton approach estimated a value of $5.8 \mathrm{~m} / \mathrm{s}$ with the true value being $5 \mathrm{~m} / \mathrm{s}$. Similarly, the estimate of the attenuation contrast converged from $440 \mathrm{~Np} / \mathrm{m}$ to $46 \mathrm{~Np} / \mathrm{m}$ where the true value was $49 \mathrm{~Np} / \mathrm{m}$.

\section{CONCLUSION}

In this work a shape based parametrization method is used in conjunction with measured ultrasound backscatter data to determine the acoustical properties and spatial structure of a simulated HIFU lesion imbedded in a tissue phantom. The measurements were obtained using a clinical linear array transducer. The ellipsoidal "lesion" presented perturbations in sound speed and attenuation only and the spatial changes in the acoustic 


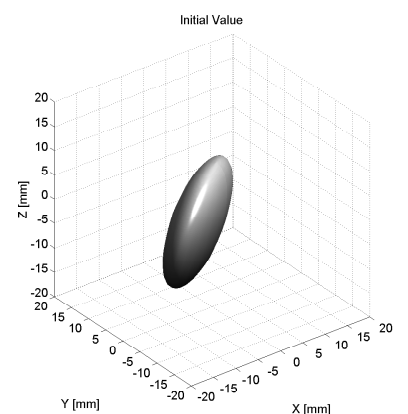

(a)

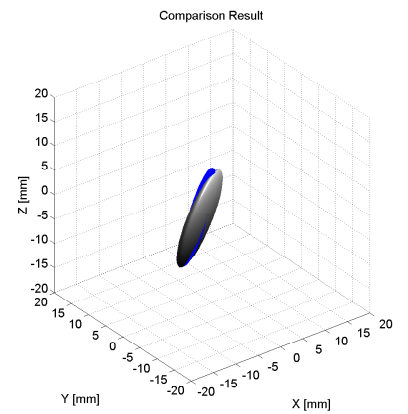

(c)

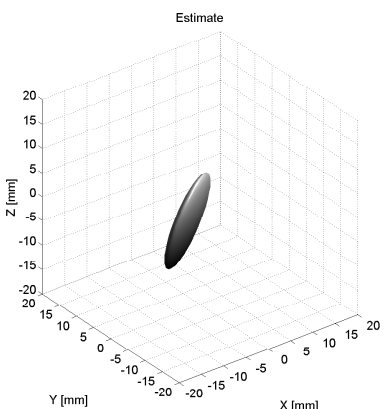

(b)

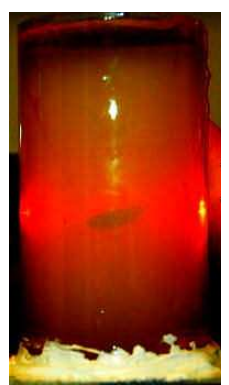

(d)

FIGURE 1. The inversion of experimental data to form an image: (a) initial value (b) final estimate (c) comparison (d) enhanced photograph of the phantom

properties were enclosed within a single ellipsoid. We were able to reconstruct the geometry of the ellipsoid and the contrasts accurately. This preliminary work indicates that, a linearized physics based model, coupled with a shape based inversion routine, provides a feasible method to monitor the progress of HIFU therapy using scattered ultrasound.

\section{ACKNOWLEDGMENTS}

This work is supported in part by CenSSIS, (NSF Award No. EEC-9986821) and NSF Award No. 0208548. The authors are grateful to Prof. Charles Thomas for technical assistance in setting up the experiments.

\section{REFERENCES}

1. P. M. Morse, and K. U. Ingard, Theoretical Acoustics, Princeton University Press, Princeton, N. J, 1986.

2. B. U. Karbeyaz, Modeling and Shape Based Inversion for Frequency Domain Ultrasonic Monitoring of Cancer Treatment, Ph.D. thesis, Northeastern University, Boston MA (2005).

3. B. U. Karbeyaz, E. L. Miller, R. O. Cleveland, and R. A. Roy, J. Acoust. Soc. Am. 114, 2379 (2003).

4. M. H. W. Philip E. Gill, Walter Murray, Practical optimization, New York : Academic Press, 1981.

5. C. Lafon, P. J. Kaczkowski, S. Vaezy, M. Noble, and O. A. Sapozhnikov, IEEE Ultrasonics Symposium Proceedings pp. 1295-1298 (2001). 